\title{
Short Range Correlations in Nuclei
}

\author{
L.B. Weinstein \\ Physics Dept, Old Dominion University, Norfolk, VA, 23529
}

\begin{abstract}
Short range correlations (SRC) are an extremely important part of nuclear structure. They are responsible for the high momentum part of the nuclear wavefunction. Instantaneous densities can significantly exceed the average neutron star density. Recent $\left(\mathrm{e}, \mathrm{e}^{\prime}\right)$ measurements at Jefferson Lab have shown that SRC are universal in nuclei from deuterium to gold, that the probability of two-nucleon SRC is 5-25\%, and that the probability of three-nucleon SRC is less than $1 \%$. Recent $\left(\mathrm{e}, \mathrm{e}^{\prime} \mathrm{pn}\right)$ measurements have measured the SRC probabilities as a function of proton momentum and have measured the joint $N N$ momentum distributions.
\end{abstract}

Keywords: short range correlations, electron scattering

PACS: $21.45 .+\mathrm{v}, 25.30 . \mathrm{Dh}$

\section{INTRODUCTION}

Two of the fundamental problems of nuclear physics are 1) to understand why nucleons remain nucleons in the nucleus and, 2) to understand precisely the structure of the nucleus in terms of nucleons and, more specifically, to understand the momentum distribution of nucleons in the nucleus. The momentum distributions of single nucleons are reasonably well measured but the joint momentum distributions of nucleon pairs are not.

The independent particle model, that describes the motions of the nucleons in the nucleus by representing the sum of their interactions by a mean field, is a surprisingly good approximation. However, because it ignores short-range nucleon-nucleon $(N N)$ interactions, it only describes nucleon momentum distributions up to $p \leq p_{\text {fermi }} \approx 220$ $\mathrm{MeV} / \mathrm{c}$.

Recent inclusive electron scattering, $A\left(e, e^{\prime}\right)$, measurements [? ? ] and theoretical calculations [? ? ? ] indicate that the high momentum part of the momentum distribution is due to short-range $N N$ interactions and therefore it has the same shape in all nuclei. They also find that there is about a five times higher probability per-nucleon to find an $N N$ pair with large relative momentum and small total momentum (i.e.: in a short range correlation) in nuclei $(A \geq 12)$ than in deuterium.

We do not understand why nucleons remain nucleons in the nucleus and why nucleons are an appropriate degree of freedom for building nuclear models. Since nucleons in short range correlations (SRC) overlap each other (however briefly), this is an excellent regime in which to study cold, high density nuclear matter. It is also the ideal place to search for nucleon modification in nuclei, since nucleons are mostly likely to be modified when their quark 'bags' overlap. Studying nucleons in this extreme regime should help us understand why nucleons remain nucleons.

\footnotetext{
CP870, Intersections of Particle and Nuclear Physics: $9^{\text {th }}$ Conference, edited by T. M. Liss

(c) 2006 American Institute of Physics 978-0-7354-0368-0/06/\$23.00
} 


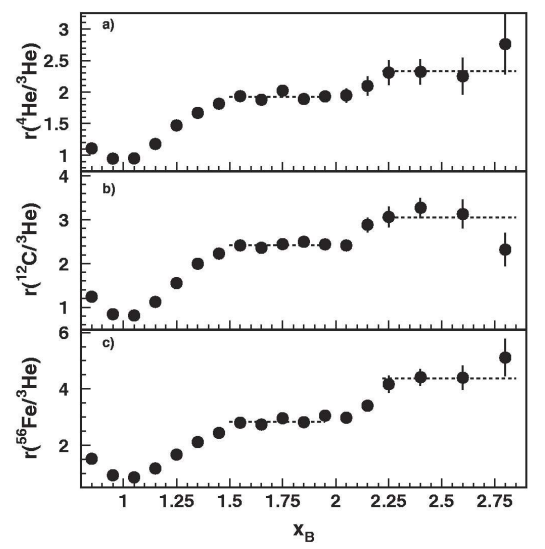

FIGURE 1. Cross section ratios of (a) ${ }^{4} \mathrm{He}$, (b) ${ }^{12} \mathrm{C}$, and (c) ${ }^{56} \mathrm{Fe}$ to ${ }^{3} \mathrm{He}$ as a function of $x_{B}$ for $Q^{2}>1.4$ $\mathrm{GeV}^{2}$. The horizontal dashed lines indicate the $N N$ and $3 N$ scaling regions.

\section{INCLUSIVE $A\left(e, e^{\prime}\right)$ MEASUREMENTS}

Inclusive electron scattering, $A\left(e, e^{\prime}\right)$, in the quasielastic region has been used to study the behavior of single nucleons in nuclei. In the nonrelativistic Plane Wave Impulse Approximation (PWIA), the cross section is proportional to the integral over the perpendicular momentum distribution of the struck nucleon. However, in reality, extracting momentum distributions from these cross sections is somewhat ambiguous and difficult.

We can however, learn about relative momentum distributions in different nuclei by measuring ratios of cross sections. The ratios of inclusive electron scattering cross sections of ${ }^{4} \mathrm{He},{ }^{12} \mathrm{C}$, and ${ }^{56} \mathrm{Fe}$ to ${ }^{3} \mathrm{He}$ have been measured at $1 \leq x_{B} \leq 3$ and $1 \leq Q^{2} \leq 3$ $\mathrm{GeV}^{2}$ in the Jefferson Lab CLAS detector [?] (where $x_{B}=Q^{2} / 2 m v, Q^{2}$ is the square of the four-momentum transfer, $v$ is the energy transfer and $m$ is the nucleon mass). At $Q^{2}>1.4 \mathrm{GeV}^{2}$, the ratios exhibit two separate plateaus, at $1.5 \leq x_{B} \leq 2$ and at $x_{B}>2.25$ (see Fig. 1. The first plateau had been seen before[? ? ] and indicates the dominance of two nucleon correlations at $p>275 \mathrm{MeV} / \mathrm{c}$. The second plateau indicates the dominance of $3 N$ correlations at $p>500 \mathrm{MeV} / \mathrm{c}$ where this momentum is balanced by the momentum of two other nucleons. The probability of $N N$ correlations ranges from $5 \%$ in deuterium to $25 \%$ in iron. The probability of $3 N$ correlations ranges from $0.2 \%$ for ${ }^{3} \mathrm{He}$ to $0.8 \%$ for ${ }^{56} \mathrm{Fe}$.

Further $A\left(e, e^{\prime}\right)$ measurements have been carried out in Hall C at JLab[? ] at $0.5 \leq$ $x_{B} \leq 2.5$ and $Q^{2} \leq 10 \mathrm{GeV}^{2}$ in order to examine scaling, momentum distributions, SRC and other topics, including the EMC effect, duality, transparency and structure functions. Preliminary results are consistent with the ratios measured in the CLAS. 

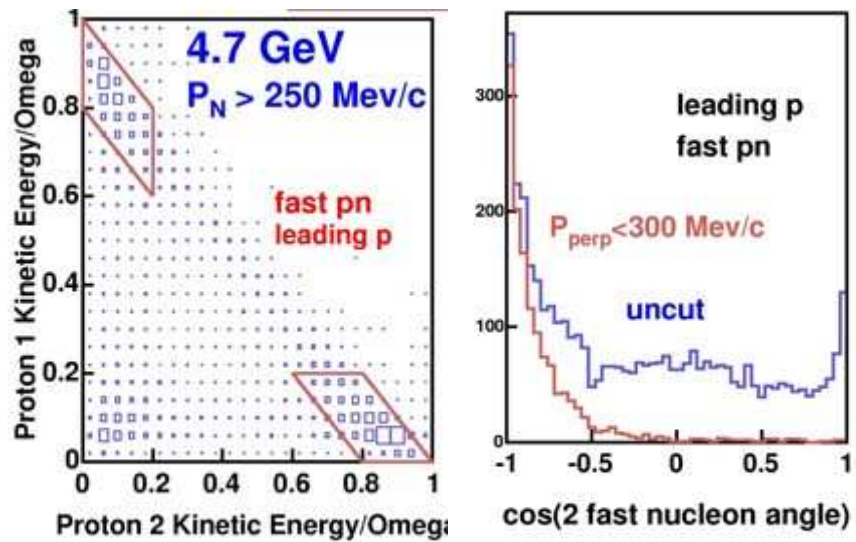

FIGURE 2. a) Energy distribution (Dalitz plot) for ${ }^{3} \mathrm{He}\left(e, e^{\prime} p p\right) n$. The kinetic energy of the first proton divided by the energy transfer is plotted versus the same quantity for the second proton. All three nucleons have momenta $p>250 \mathrm{MeV} / \mathrm{c}$. b) Opening angle of the two non-leading nucleons. Note the peak at $\cos \theta_{p n}=-1$. The lower curve is cut on the perpendicular momentum (relative to the momentum transfer direction) of the leading proton $p_{\perp}<300 \mathrm{MeV} / \mathrm{c}$. Note that the two non-leading nucleons are almost entirely back-to-back.

\section{EXCLUSIVE $A\left(e, e^{\prime} p N\right)$ MEASUREMENTS}

While inclusive $A\left(e, e^{\prime}\right)$ measurements can provide information on the probability of finding SRC in nuclei, we need to measure the ejected nucleons in order to learn about the momentum distrbutions of the correlated pairs.

${ }^{12} \mathrm{C}\left(e, e^{\prime} p\right),\left(e, e^{\prime} p n\right)$, and $\left(e, e^{\prime} p p\right)$ was recently measured in Jefferson Lab Hall $\mathrm{A}[?$ ]. The electron and first proton were measured in the High Resolution Spectrometers and the second nucleon was measured in BigBite or the new low energy neutron detector. The measurement was performed at $Q^{2}=2 \mathrm{GeV}^{2}$ and $x_{B}=1.23$ to reduce the contributions from Meson Exchange Currents (MEC) and Isobar Configurations (IC). Very preliminary initial results show that the higher the missing momentum of the $\left(e, e^{\prime} p\right)$ reaction ( $\vec{p}_{\text {miss }}=\vec{q}-\vec{p}$ where $\vec{p}$ is the momentum of the detected proton), the greater the probability that a second proton was also emitted. This is consistent with the SRC model.

A difficulty with most $\left(e, e^{\prime} p N\right)$ measurements designed to study SRC is that the virtual photon interacts with the correlated $N N$ pair. This interaction is a coherent sum of one-body currents (impulse approximation or single nucleon knockout) and two-body currents (Final State Interactions [FSI], MEC and IC). In order to study the momentum distributions of the pair without a tremendous amount of theoretical ambiguity, we need to separate out the one-body currents.

This was done in fully exclusive ${ }^{3} \mathrm{He}\left(e, e^{\prime} p p\right) n$ measurements in CLAS with 2.2 $\mathrm{GeV}[?]$ ] and $4.7 \mathrm{GeV}$ electron beams. Figure 2a shows the energy distribution (Dalitz plot) for the reaction at $4.7 \mathrm{GeV}$ for nucleons with momenta $p>250 \mathrm{MeV} / \mathrm{c}$. Note that the distribution is dominated by peaks where one of the nucleons carries away most 
of the transferred energy (the 'leading' nucleon) and the remaining two nucleons each carry less than $20 \%$ of the transferred energy. The opening angle of the two non-leading nucleons (see Fig. 2b) has a significant back-to-back peak $\left(\cos \theta_{p n}=-1\right)$. When we cut on the perpendicular momentum (relative to $\vec{q}$ ) of the leading nucleon, $p_{\perp}<300 \mathrm{MeV} / \mathrm{c}$, then the two non-leading nucleons are almost entirely back-to-back (see the lower curve in Fig. 2b).

This indicates that we have measured SRC by knocking out one nucleon and detecting the spectator correlated pair as it flew apart. Further evidence for this comes from calculations by Golak[? ] which include knockout of the leading nucleon plus the reinteraction of the spectator pair with each other (i.e.: it does not include final state interactions between the knocked out nucleon and the spectator pair). These calculations describe the magnitude of the cross section (to within $15 \%)$ at both $2.2 \mathrm{GeV}\left(0.5<Q^{2}<\right.$ $\left.1 \mathrm{GeV}^{2}\right)$ and $4.7 \mathrm{GeV}\left(1.5<Q^{2}<2 \mathrm{GeV}^{2}\right)$ and for both $p p$ and $p n$ spectator pairs. It also describes qualitatively the spectator pair angles and relative and total momentum distributions.

Thus, these exclusive experiments are providing the first measurements of correlated pair momentum distributions in nuclei.

\section{ACKNOWLEDGMENTS}

The author gratefully acknowledges John Arrington, Donal Day, and Doug Higinbotham for providing preliminary data from their measurements. The author thanks the United States Department of Energy for support under contract DE-FG02-96ER40960. The Southeastern Universities Research Association (SURA) operates the Thomas Jefferson National Accelerator Facility for the United States Department of Energy under contract DE-AC05-84ER40150. 